\title{
Is Observation Dead in Follicular Lymphoma? No, But the Apoptosis Pathway Has Been Activated
}

\author{
Richard I. Fisher, MD, and Nadia Khan, MD
}

\begin{abstract}
Follicular lymphoma (FL), the most common indolent type of non-Hodgkin's lymphoma, presents with a highly variable clinical course and affects the overall survival (OS) of patients. Although observation has been adopted widely by clinicians in the management of patients with $\mathrm{FL}$, the benefits of early treatment must be reviewed in light of the significant progress made in the treatment of symptomatic or higher-tumor-burden FL. When treatment is indicated, a variety of combination chemotherapeutic regimens have proven efficacy and have shown improvements in both progression-free and event-free survival, and the addition of rituximab to these regimens has shown a statistically significant improvement in OS. Additionally, single-agent rituximab has been added to the possible therapeutic options for patients with lowtumor-burden FL. Although a paucity of clinical data exists on the upfront treatment of the low-tumor-burden population, the question of whether early treatment, especially with the newer rituximab-containing chemotherapy regimens, would improve OS is thought-provoking. Furthermore, novel targeted therapies with tolerable side effect profiles are rapidly advancing in the treatment of non-Hodgkin's lymphoma. The future for patients with low-tumor-burden FL is brighter than ever before. (J Natl Compr Canc Netw 2015;13:363-366)
\end{abstract}

\section{NCCN: Continuing Education}

\section{Accreditation Statement}

This activity has been designated to meet the educational needs of physicians and nurses involved in the management of patients with cancer. There is no fee for this article. No commercial support was

From Fox Chase Cancer Center, Philadelphia, Pennsylvania. Submitted April 30, 2014; accepted for publication September 18, 2014. Dr. Khan has disclosed that she is a member of the speakers' bureau for Genentech, Inc. Dr. Fisher had disclosed that he is a consultant for Johnson \& Johnson Services, Inc.; MorphoSys AG; and Celgene Corporation.

Correspondence: Nadia Khan, MD, Fox Chase Cancer Center, 333 Cottman Avenue, Philadelphia, PA 19111. E-mail: Nadia.Khan@fccc.edu

\section{EDITOR}

Kerrin M. Green, MA, Assistant Managing Editor, JNCCN-Journal of the National Comprehensive Cancer Network

Ms. Green has disclosed that she has no relevant financial relationships. received for this article. The National Comprehensive Cancer Network (NCCN) is accredited by the ACCME to provide continuing medical education for physicians.

NCCN designates this journal-based CME activity for a maximum of 1.0 AMA PRA Category 1 Credit(s) ${ }^{\mathrm{TM}}$. Physicians should claim only the credit commensurate with the extent of their participation in the activity.

NCCN is accredited as a provider of continuing nursing education by the American Nurses Credentialing Center's Commission on Accreditation.

This activity is accredited for 1.0 contact hour. Accreditation as a provider refers to recognition of educational activities only; accredited status does not imply endorsement by NCCN or ANCC of any commercial products discussed/displayed in conjunction with the educational activity. Kristina M. Gregory, RN, MSN, OCN, is our nurse planner for this educational activity.

All clinicians completing this activity will be issued a certificate of participation. To participate in this journal CE activity: 1) review the learning objectives and author disclosures of both articles; 2) study the education content of both articles; 3 ) take the posttest with a $66 \%$ minimum passing score and complete the evaluation at http:// education.nccn.org/node/62684; and 4) view/print certificate.

Release date: March 6, 2015; Expiration date: March 6, 2016

\section{Learning Objectives}

Upon completion of this activity, participants will be able to:

- Review the pros and cons of early treatment versus observation in the management of patients with $\mathrm{FL}$

- Discuss the potential benefits of chemoimmunotherapy as initial treatment for the management of patients with $\mathrm{FL}$

- Identify the indications of therapy in patients requiring treatment for $\mathrm{FL}$

\section{CE AUTHORS}

Deborah J. Moonan, RN, BSN, Director, Continuing Education, has disclosed that she has no relevant financial relationships.

Ann Gianola, MA, Manager, Continuing Education Accreditation \& Program Operations, has disclosed that she has no relevant financial relationships.

Kristina M. Gregory, RN, MSN, OCN, Vice President, Clinical Information Operations, has disclosed that she has no relevant financial relationships. Rashmi Kumar, PhD, Senior Manager, Clinical Content, has disclosed that she has no relevant financial relationships.

Hema Sundar, PhD, Oncology Scientist/Senior Medical Writer, has disclosed that she has no relevant financial relationships. 
Fisher and Khan

The concept of observation, or the "watch and wait" approach, was originally proposed in 1979 by Portlock and Rosenberg ${ }^{1}$ at Stanford University, when they reported results on 44 patients who, for various reasons, had not received initial therapy after being diagnosed with follicular lymphoma (FL). The reasons for the decision not to treat were complex and not entirely evident on review, but included assessment by the treating oncologists that these patients presented with a benign or indolent disease course, and that it was the patients' personal choice to defer treatment. Portlock and Rosenberg were also impressed with the toxicity associated with the chemotherapy administered to these patients in the absence of clear evidence of long-term benefit, at least from a survival perspective. At that time, and for many years after, the median survival of patients with newly diagnosed, advanced-stage FL ranged from 5 to 7 years, with no evidence of a plateau in the survival curve (ie, no suggestion that any patients were cured). A rarely considered and little known fact is that the original Stanford policy of watch and wait permitted local radiation therapy to be administered in up to 3 different locations.

Over the years, most physicians treating FL adapted the concept of watch and wait, although the criteria for selection of this treatment have varied somewhat. In general, watch and wait was the preferred therapy for newly diagnosed patients who presented with low tumor burden and no symptoms of FL. Instead of defining the criteria for watch and wait, some groups have defined the criteria for treatment, such as the Groupe d'Etudes des Lymphomes Folliculaires (GELF) criteria. ${ }^{2}$ More recent randomized trials have confirmed that the watch and wait approach has comparable survival to initial treatment with single-agent alkylating agents, such as chlorambucil. A major advantage of this approach was that it afforded the patient a reasonably long period (2.5-3.0 years) of treatment-free survival, based on the results of retrospective studies in the pre-rituximab era. ${ }^{3}$

Fortunately, much has changed since the initial reports of watch and wait in the 1970s. A major advancement in the treatment of FL was the introduction of the monoclonal antibody rituximab. As a single agent, rituximab maintenance produced an $84 \%$ overall response rate, and at 3 years, $88 \%$ of patients on the maintenance arm still did not require therapy, compared with $46 \%$ of those undergoing watchful waiting. ${ }^{4}$ Furthermore, it is extremely well tolerated, with very little of the toxicity associated with conventional chemotherapy. It is well-known by lymphoma experts that there is a subset of patients with disease with a median survival of 5 to 7 years for whom the concept of watch and wait is unacceptable and associated with significant psychological stress; the size of this group has never been accurately characterized. Thus, single-agent rituximab now provides practitioners and patients with a well-tolerated and effective initial treatment to consider as an alternative to watch and wait, while prolonging the time to chemotherapy.

However, the combination of rituximab with conventional chemotherapy offers insight into the potential benefits of initial therapy for the low-tumorburden population. Until the past decade, no evidence showed that different initial therapies for FL could impact overall survival (OS). However, recent studies of rituximab in combination with chemotherapy have clearly shown that this is no longer true. The study by Marcus et $\mathrm{al}^{5}$ randomized previously untreated patients who required initial therapy to either CVP (cyclophosphamide, vincristine, and prednisone) and R-CVP (cyclophosphamide, vincristine, and prednisone with rituximab). The R-CVP group showed improvement in not only failure-free survival (FFS) but also OS ( $83 \%$ vs $77 \% ; P=.03$ ). Similarly designed studies from the German Low Grade Lymphoma Study Group (GLSG) compared CHOP (cyclophosphamide, doxorubicin, vincristine, and prednisone) with R-CHOP (cyclophosphamide, doxorubicin, vincristine, and prednisone with rituximab). Again, FFS and $O S$ were improved (median overall response rate, $96 \%$ vs $90 \% ; P=.01){ }^{6}$

In 2005, a study reported the survival of patients with previously untreated FL treated on a sequential series of treatment approaches by the SWOG Lymphoma Committee spanning 3 decades. ${ }^{7}$ The survival of patients treated with $\mathrm{R}-\mathrm{CHOP}$ was superior to that of those observed on prior treatment regimens. Finally, data derived from the SEER database showed that the survival of patients with FL had improved dramatically on a population basis, from the time period of 1979 to $1999 .{ }^{8}$ In the most recent studies reported by Press et $\mathrm{al}^{9}$ for SWOG, the 5-year PFS rate of patients with FL treated with immunochemotherapy was $60 \%$ or higher and the 5 -year OS was $86 \%$, but approaches $80 \%$ at 10 years for patients treated with $\mathrm{R}-\mathrm{CHOP}$. 
Observation in Follicular Lymphoma: Not Yet

Clearly, patients with newly diagnosed FL now have a dramatically better prognosis than 20 years ago, when $50 \%$ died by 5 to 7 years.

This improvement in the ability to allow patients with FL to enjoy prolonged initial remissions is evident in the PRIMA study. ${ }^{10}$ In this study, Salles et $\mathrm{al}^{10}$ showed that maintenance therapy with rituximab for 2 years after initial rituximab in combination with chemotherapy resulted in prolonged progression-free survival, although OS was not impacted. Notably, centers participating in the PRIMA study had to select one combination rituximab/chemotherapy regimen for use in all patients entering the trial; approximately 75\% chose R-CHOP, 20\% chose R-CVP, and only 5\% chose a fludarabine-based regimen. A retrospective analysis clearly showed that the patients who received $\mathrm{R}-\mathrm{CHOP}$ had superior survival compared with those receiving R-CVP; too few patients were treated with fludarabine-based regimens to analyze. These data suggested that in the combination rituximab/chemotherapy era, intensity of treatment, which also resulted in higher complete response rates for the $\mathrm{R}-\mathrm{CHOP}$ group, might matter.

Bendamustine, a bifunctioning alkylating agent, has demonstrated efficacy in the treatment of FL and has emerged as part of frontline therapy in the treatment of patients with FL. The first randomized study to compare R-CHOP versus bendamustine with rituximab (BR) was the StiL study. ${ }^{11}$ Patients with advanced-stage FL had similar OS, whether treated with R-CHOP or $\mathrm{BR}$, and, notably, median progression-free survival was superior in the BR arm compared with R-CHOP (70 vs 31 months; $P<.0001$ ). Subsequently, the BRIGHT study showed that BR is noninferior to $\mathrm{R}-\mathrm{CHOP}$ in patients with advanced FL. ${ }^{12}$ Given the largely acceptable toxicity profile of $\mathrm{BR}$, it has emerged as a preferred therapeutic option for both upfront and relapsed disease, by many clinicians.

Finally, it is important to consider whether the other well-known paradigm describing FL, that "it is an incurable disease," is still valid. How do we define cure? Understanding that no data show that patients with FL have the same survival as a matched population without this lymphoma, several other surrogates can be evaluated to demonstrate curability. If a significant number of patients with a given disease do not experience recurrence after initial therapy and die from other unrelated causes, does that observation suggest some patients are being cured? It is well-known that the uncommon patient with localized FL treated with radiation therapy can have extraordinarily good 5- and 10-year FFS and OS. Patients with newly diagnosed FL present at an average age of 60 years. Median 5-year progression-free survival for patients in this age group who have low Follicular Lymphoma International Prognostic Index-2 scores now approaches $80 \%$. Thus, considering the ability to attain initial remissions exceeding 10 years with therapy, are some patients with FL now considered cured? If one accepts the argument that some patients with FL are now cured with rituximabcontaining regimens, does that change the accepted concept of watch and wait? What curable diseases are permitted to go untreated for a prolonged period?

In summary, initial observation, or watch and wait, has been a standard approach to treating asymptomatic patients with FL with a low tumor burden for several decades. It provides patients with an opportunity to avoid treatment for a significant period. However, most patients will require therapy at some point. Recall that watch and wait was never shown to be superior to initial therapy. Single-agent rituximab therapy now provides an alternative for that same population, especially those who are not comfortable with the concept of withholding treatment for a malignant and ultimately fatal disease. If one accepts the potential curability of some patients with FL, does that change one's willingness to delay treatment?

Furthermore, much excitement currently surrounds the new targeted oral agents being developed for the treatment of B-cell lymphomas. Impressive single-agent activity has been seen in relapsed/refractory FL treated with the newer kinase inhibitors. Will these inhibitors replace rituximab-containing chemotherapy or, as an adjunct, will they make rituximab-containing chemotherapy even more active? These studies are ongoing and the positive impact that they will have on treatment strategies for FL are eagerly anticipated. Further future stratification of patients according to molecular and clinical features may allow clinicians to determine which patients with low tumor burden are likely to benefit from an upfront strategy.

Old paradigms die hard in medicine, such as "observation is the only reasonable treatment for asymptomatic patients with low-tumor-burden FL"; "OS is not an end point that can be used in studies of FL"; and "FL is noncurable." In many ways, these paradigms delay the goal of scientific investigation into 
Fisher and Khan

the treatment of an ultimately fatal malignancy, and the ultimate goal to cure the disease with reasonable therapy.

Observation may not be dead, but upfront therapy for FL remains an appropriate strategy. Although for now the debate over the best initial treatment approach in FL continues, this will likely not be a debatable subject 10 years from now.

\section{References}

1. Portlock CS, Rosenberg SA. No initial therapy for stage III and IV nonHodgkin's lymphomas of favorable histologic types. Ann Intern Med 1979;90:10-13.

2. Brice $\mathrm{P}$, Bastion $\mathrm{Y}$, Lepage $\mathrm{E}$, et al. Comparison in low-tumor-burden follicular lymphomas between an initial no-treatment policy, prednimustine, or interferon alfa: a randomized study from the Groupe d'Etude des Lymphomes Folliculaires. Groupe d'Etude des Lymphomes de l'Adulte. J Clin Oncol 1997;15:1110-1117.

3. Ardeshna KM, Smith P, Norton A, et al. Long-term effect of a watch and wait policy versus immediate systemic treatment for asymptomatic advanced-stage non-Hodgkin lymphoma: a randomised controlled trial. Lancet 2003;362:516522

4. Ardeshna KM, Qian W, Smith P, et al. Rituximab versus a watch-andwait approach in patients with advanced-stage, asymptomatic, non-bulky follicular lymphoma: an open-label randomised phase 3 trial. Lancet Oncol 2014;15:424-435.
5. Marcus R, Imrie K, Solal-Celigny P, et al. Phase III study of R-CVP compared with cyclophosphamide, vincristine, and prednisone alone in patient with previously untreated advanced follicular lymphoma. J Clin Oncol 2008;26:4579-4586

6. Hiddemann W, Kneba M, Dreyling M, et al. Frontline therapy with rituximab added to the combination of cyclophosphamide, doxorubicin, vincristine, and prednisone (CHOP) significantly improves the outcome for patients with advanced-stage follicular lymphoma compared with therapy with $\mathrm{CHOP}$ alone: results of a prospective randomized study of the German Low-Grade Lymphoma Study Group. Blood 2005;106:3725-3732.

7. Fisher RI, LeBlanc M, Press OW, et al. New treatment options have changed the survival of patients with follicular lymphoma. J Clin Oncol 2005;23:84478452 .

8. Swenson WT, Wooldridge JE, Lynch CF, et al. Improved survival of follicular lymphoma patients in the United States. J Clin Oncol 2005;23:5019-5026.

9. Press OW, Unger JM, Rimsza LM, et al. Phase III randomized intergroup trial of CHOP plus rituximab compared with CHOP chemotherapy plus (131)iodinetositumomab for previously untreated follicular non-Hodgkin lymphoma: SWOG S0016. J Clin Oncol 2013;31:314-320.

10. Salles G, Seymour JF, Offner F, et al. Rituximab maintenance for 2 years in patients with high tumour burden follicular lymphoma responding to rituximab plus chemotherapy (PRIMA): a phase 3, randomised controlled trial. Lancet 2011;377:42-51

11. Rummel MJ, Niederle $N$, Maschmeyer $G$, et al. Bendamustine plus rituximab versus CHOP plus rituximab as first-line treatment for patients with indolent and mantle-cell lymphomas: an open-label, multicentre, randomised, phase 3 non-inferiority trial. Lancet 2013;381:1203-1210.

12. Flinn IW, van der Jagt R, Kahl BS, et al. Randomized trial of bendamustinerituximab or R-CHOP/R-CVP in first-line treatment of indolent NHL or MCL: the BRIGHT study. Blood 2014;123:2944-2952.

\section{Instructions for Completion}

To participate in this journal CE activity: (1) review the learning objectives and author disclosures of both articles; (2) study the education content of both articles; 3 ) take the posttest with a $66 \%$ minimum passing score and complete the evaluation at http://education.nccn.org/node/62684; and 4) view/print certificate. After reading the article, you should be able to answer the following multiple-choice questions. Credit cannot be obtained for tests completed on paper. You must be a registered user on NCCN.org. If you are not registered on NCCN.org, click on "New Member? Sign up here" link on the left hand side of the Web site to register. Only one answer is correct for each question. Once you successfully answer all posttest questions you will be able to view and/or print your certificate. Software requirements: Internet.

\section{Posttest Questions}

1. True or False: Initial observation, or "watch and wait," is an appropriate strategy for selected patients with FL.

2. In a retrospective analysis of the PRIMA study, which regimen clearly showed superior survival benefit?

a. Fludarabine-based regimen
b. R-CVP
c. R-CHOP
d. None of the above

3. True or False: Bendamustine and rituximab is noninferior to RCHOP in patients with advanced FL.

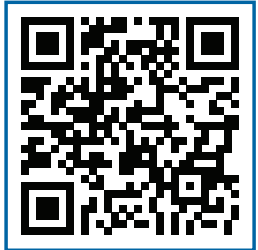

